\title{
Acylcarnitine to Free Carnitine Ratio Measurement
}

National Cancer Institute

\section{Source}

National Cancer Institute. Acylcarnitine to Free Carnitine Ratio Measurement. NCI

Thesaurus. Code C147289.

The determination of the ratio of acylcarnitine compared to free carnitine present in a sample. The measurement may be expressed as a ratio or percentage. 